Pacific Journal of Mathematic 


\title{
ON THE EQUIVALENCE OF TWO TYPES OF OSCILLATION FOR ELLIPTIC OPERATORS
}

\author{
W. Allegretto
}

\begin{abstract}
The strong oscillation of a second order symmetric elliptic operator is shown to be equivalent to the oscillation of all solutions of the associated homogeneous equation. Extensions of a nonoscillation theorem and of an existence theorem are obtained as applications.
\end{abstract}

Introduction. Let $\left\{E^{n}, \infty\right\}$ denote the topological space formed by the standard one point compactification of $n$-dimensional Euclidean space $E^{n}$. A real valued function $u$ with domain in $E^{n}$ is said to be oscillatory $($ at $\infty$ ) iff $\infty$ belongs to the closure (in the topology of $\left.\left\{E^{n}, \infty\right\}\right)$ of the set $\left\{x \mid x \in E^{n}\right.$ and $\left.u(x)=0\right\}$. Let $L$ denote a second order symmetric elliptic operator with coefficients defined in an unbounded domain $\Omega$ of $E^{n}$. Following I. Glazman [6], we define $L$ to be strongly oscillatory (at $\infty$ ) iff $L$ has a nodal domain in $N \cap \Omega$ for any given neighborhood $N$ of $\infty$. That is: Given any neighborhood $N$ of $\infty$, there exists a bounded domain $D \subset N \cap \Omega$ for which zero is the smallest eigenvalue for $L$ (corresponding to Dirichlet boundary conditions). Since the classical Sturm-Kneser theorem can be extended to partial differential equations by means of the Swanson-Picone identity, [14, p. 187], [2], it follows that if $L$ is strongly oscillatory then every $C^{2}$ function $v$ which is a solution of the equation $L u=0$ in $N \cap \Omega$, for some neighbourhood $N$ of $\infty$, must be oscillatory. This connection between the strong oscillation of $L$ and the oscillation of the solutions to the equation $L u=0$ has been noted for some time, beginning with results of $\mathrm{K}$. Kreith [8], for special cases of $L$; Headley and Swanson [7], for the general case; and, more recently, several other authors. Further extensions of these concepts have also been made to the case of elliptic systems [1], [16] and eigenvalue problem [2], [3]. We refer the reader to the recent book by K. Kreith [9], where these ideas are discussed and an extensive bibliography is given.

It is our main purpose to show that the strong oscillation of a second order elliptic operator $L$ is equivalent to the oscillation of all solutions $u$ of the equation $L u=0$ in neighborhoods of $\infty$, if the coefficients of $L$ and $\Omega$ are reasonably regular. This extends a result which is obviously true for ordinary differential equations. As applications of our results, a nonoscillation theorem of C. A. Swanson is strengthened and some related results of L. M. Kuks are clarified and extended. 
We shall restrict our discussion to the case where $\Omega$ is an unbounded domain and $L$ has regular coefficients. The analogues of our results for the case where $\Omega$ is bounded but the coefficients of $L$ are singular at some boundary point of $\Omega$ will be obvious from the presentation.

2. Assumptions and main results. As is usual, points of $E^{n}$ will be denoted by $x=\left(x_{1}, \cdots, x_{n}\right)$ and differentiation with respect to $x_{i}$ by $D_{i}$ for $i=1, \cdots, n$. Let $\Omega$ denote an unbounded domain of $E^{n}$. We shall use the following notation throughout:

$$
\begin{aligned}
& \Omega_{\rho_{1}, \rho}=\Omega \cap\left\{x \mid x \in E^{n} \text { and } \rho_{1}<|x|<\rho\right\}, \\
& \Omega_{\rho, \infty}=\Omega \cap\left\{x \mid x \in E^{n} \text { and } \rho<|x|\right\},
\end{aligned}
$$

where $0<\rho_{1}<\rho<\infty$.

Let $L$ denote the elliptic operator formally given by:

$$
L u=-\sum_{i, j=1}^{n} D_{i}\left[a_{i j} D_{j} u\right]+c u, \quad a_{i j}=a_{j i} .
$$

The coefficients $a_{i j}$ are assumed of class $C^{m+1}$ and $c$ is assumed of class $C^{m}$ where $m=3[[3+n / 2] / 2]$ in the closure of any bounded subdomain of $\Omega$, where $[q]$ denotes the largest integer not exceeding $q$. $L$ is assumed uniformly elliptic in any bounded subdomain of $\Omega$. These assumptions are more restrictive than what is needed for many of the results, however they lead to a unified and simple presentation. About $\Omega$ we shall only assume that $\Omega_{\rho_{1}, \rho}$ is a domain for all $\rho_{1}, \rho$ with $\rho_{0}<\rho_{1}<\rho<\infty$ for some $\rho_{0}>0$. Unlike most results for unbounded domains, no other restrictions are placed on $\Omega$ or on the coefficients of $L$ at $\infty$ or on the sign of $c$.

Lemma 0 . Let $\beta$ be a positive constant and $x_{0}$ a point in $\Omega$ so that $\left\{x|| x-x_{0} \mid \leqq \beta\right\} \subseteq \Omega$. Define the $C_{0}^{2 l-1}(\Omega)$ function $\Psi$ as

$$
\Psi(x)=\left\{\begin{array}{ccc}
-\left(\left|x-x_{0}\right|^{2}-\beta^{2}\right)^{2 l}, & \text { if } & \left|x-x_{0}\right| \leqq \beta \\
0 & , \text { if } & \left|x-x_{0}\right| \geqq \beta
\end{array}\right.
$$

Then given $\varepsilon, 0<\varepsilon<1 / 2$, there is a $l_{0}=l_{0}\left(x_{0}, \beta, \varepsilon\right)$ such that for each positive integer $l \geqq l_{0}$, the following hold:

(i) $L \Psi(x) \geqq 0$ for $\varepsilon \beta \leqq\left|x-x_{0}\right|$

(ii) $L \Psi(x)>0$ for $\varepsilon \beta \leqq\left|x-x_{0}\right| \leqq(1-\varepsilon) \beta$.

The proof of Lemma 0 follows easily from the locally uniform ellipticity of $L$ and the local boundedness of the coefficients of $L$.

As an immediate consequence of Lemma 0 , we note that given any arbitrarily large $q>0$ and any sufficiently small $\varepsilon>0$ we can 
construct a function $\phi_{0} \in C_{0}^{2}\left(\Omega_{q, q+2 \varepsilon}\right)$ such that $L\left(\phi_{0}\right) \geqq 0(\not \equiv 0)$ in $\Omega_{q+\varepsilon, q+2 \varepsilon}$. Given any bounded subdomain $D$ of $\Omega$ we introduce the space $C^{1}(\bar{D})$ of all continuously differentiable functions in $\bar{D}$ and the space $H^{1}(D)$ which is the completion of $C^{1}(\bar{D})$ in the norm:

$$
\|u\|_{1}^{2}=\int_{D}\left\{\sum_{i=1}^{n}\left(D_{i} u\right)^{2}+u^{2}\right\} d \Omega
$$

By $H_{0}^{1}(D)$ we denote the closure in the \|\|$_{1}$-norm of the space $C_{0}^{\infty}(D)$, of all infinitely differentiable functions with compact support in $D$.

We shall not distinguish in notation between the equivalence classes which form the elements of $H^{1}(D)$ and functions chosen from various equivalence classes.

Given any function $u \in H_{0}^{1}(D)$, we define $u$ to be nonnegative in the $H_{0}^{1}$ sense iff there exists a sequence of nonnegative $C_{0}^{1}(D)$ functions which converges to $u$ in the \|\|$_{1}$-norm. If $u, v \in H_{0}^{1}(D)$, we write $u \geqq v$ iff $u-v \geqq 0$ in the $H_{0}^{1}$ sense. Let $G$ denote the function from $R^{1}$ to $R^{1}$ given by:

$$
G(x)=\left\{\begin{array}{ll}
x & x \geqq 0 \\
0 & x<0
\end{array} .\right.
$$

For any $u \in H_{0}^{1}(D)$, we set $u^{+}=G \circ u, u^{-}=G \circ(-u)$ and define $|u|$ by $|u|=u^{+}+u^{-}$.

The form $B(u, \dot{\rho})$ given by:

$$
B(u, \dot{\phi})=\int_{D} \sum_{i, j=1}^{n} a_{i j} D_{i} u D_{j} \dot{\phi}+c u \dot{\phi}
$$

is naturally associated with the operator $L$ and subdomain $D$. A function $u \in H^{1}(D)$ is called subsolution with respect to $L$ in $D$ iff $B(u, \dot{\phi}) \leqq 0$ for $\dot{\phi} \in C_{0}^{\infty}(D), \dot{\phi} \geqq 0$. A generalized solution of $L u=f$ is a function $u$ in $H^{1}(D)$ such that $B(u, \dot{\rho})=(f, \dot{\rho})$ for all $\dot{\phi} \in C_{0}^{\infty}(D)$ where, as usual, we set $(f, \dot{\phi})=\int_{D} f \dot{\varphi}$.

The above terminology was introduced in [13] where the following two results were established (in much greater generality):

Lemma 1 [13, p. 18]. If $u$ is a member of $H_{0}^{1}(D)$ then so are $u^{+}$and $u^{-}$and:

$$
D_{i} u^{+}=\left\{\begin{array}{ll}
0 & u \leqq 0 \\
D_{\imath} u & u \geqq 0
\end{array} \text { a.e.D; } \quad D_{i} u^{-}=\left\{\begin{array}{ll}
0 & u \geqq 0 \\
-D_{i} u & u \leqq 0
\end{array}\right. \text { a.e.D. }\right.
$$

Lемма $2[13, \mathrm{p} .75]$. If $u, v$ are subsolutions in $D$ and the form $B$ is coercive over $H_{0}^{1}(D)$ then $\max (u, v)$ is also a subsolution. 
If $u \in H_{0}^{1}(D)$ then clearly $u^{+}$and $u^{-}$are nonnegative in the $H_{0}^{1}(D)$ sense.

The next two lemmas show that if $L$ has no nodal domains in $\Omega_{m, \infty}$ then there exists a positive $C^{2}$ function $u$ such that $L u(x)=$ 0 for all $|x|$ sufficiently large. Such a function $u$ is obtained as the limit of solutions of suitable boundary value problems involving bounded subdomains of $\Omega_{m, \infty}$.

Lemma 3. Let $L$ have no nodal domain in $\Omega_{m, \infty}$ for some integer $m>\rho_{0}$ and let $\dot{\phi}_{0} \in C_{0}^{2}\left(\Omega_{m, m+2 \varepsilon}\right)$ be a function constructed by the above procedures (for some $\varepsilon>0$ ) such that $L \dot{\phi}_{0} \geqq 0(\not \equiv 0)$ in $\Omega_{m+\varepsilon, m+2 \varepsilon}$. Then for each integer $k>m+2 \varepsilon$ there exists a function $u_{k}$ in $H_{0}^{1}\left(\Omega_{m+\varepsilon, k}\right)$ such that $L u_{k}=L \dot{\phi}_{0}$ in a generalized sense. Furthermore, if $k_{1}>k_{2}>$ $m+2 \varepsilon$ then $u_{k_{1}} \geqq u_{k_{2}} \geqq 0$.

Proof. Since $L$ has no nodal domains in $\Omega_{m, \infty}$ then for each integer $k, k>m+2 \varepsilon$, there exists a positive constant $\gamma$ such that for all functions $\phi \in C_{0}^{\infty}\left(\Omega_{m+\varepsilon, k}\right)$ we have:

$$
(L \dot{\phi}, \dot{\phi}) \geqq \gamma(\dot{\phi}, \dot{\phi}) \text {. }
$$

Consequently $L$ is uniformly positive definite in $C_{0}^{\infty}\left(\Omega_{m+\varepsilon, k}\right)$ and we can form the Friedrichs extension of $L$ (also denoted by $L$ ) whose domain is contained in the completion of $C_{0}^{\infty}\left(\Omega_{m+\varepsilon, l}\right)$ in the \|\|$_{L}$-norm, where $\|\dot{\phi}\|_{L}^{2}=(\dot{\phi}, L \dot{\varphi})$. We note that if $\dot{\phi} \in C_{0}^{\infty}\left(\Omega_{m+\varepsilon, k}\right)$ then $\|\dot{\phi}\|_{L}^{2} \leqq$ $M\|\dot{\rho}\|_{1}^{2}$ for some constant $M$ which depends on the coefficients of $L$, and, conversely,

$$
\|\dot{\varphi}\|_{1}^{2} \leqq\left[\frac{1}{\lambda}\left(1+\frac{N}{\gamma}\right)+\frac{1}{\gamma}\right]\|\dot{\rho}\|_{L}^{2}
$$

where $\lambda$ denotes a positive lower bound on the smallest eigenvalue of $\left(a_{i j}(x)\right)$ and $N=\sup |\mathrm{c}(x)|$ for $x \in \Omega_{m+\varepsilon, k}$. Consequently, the \|\|$_{L^{-}}$ norm and \|\|$_{1}$-norm are equivalent for $C_{0}^{\infty}\left(\Omega_{m+\varepsilon, k}\right)$ and the completion of $C_{0}^{\infty}\left(\Omega_{m+\varepsilon, k}\right)$ in the \|\|$_{L}$-norm is $H_{0}^{1}\left(\Omega_{m+\varepsilon, k}\right)$. By the results stated, for example, in [12, Chapter 1] there exists a unique function $u_{k}$ in $H_{0}^{1}\left(\Omega_{m+\varepsilon, k}\right)$ which is a generalized solution of $L u_{k}=L \dot{\phi}_{0}$ in $\Omega_{m+\varepsilon, k}$ and is further characterized as the function which minimizes the functional:

$$
J(\phi)=\|\dot{\phi}\|_{L}^{2}-2\left(\dot{\phi}, L \dot{\phi}_{0}\right)
$$

over the space $H_{0}^{1}\left(\Omega_{m+\varepsilon, k}\right)$. By Lemma 1, it follows that $\left|u_{k}\right| \in H_{0}^{1}\left(\Omega_{m+\varepsilon, k}\right)$, $\left(D_{i}\left|u_{k}\right|\right)^{2}=\left(D_{i} u_{k}\right)^{2}$, and $\left|u_{k}\right|^{2}=u_{k}^{2}$ a.e. $\Omega_{m+\varepsilon, k}$. Consequently,

$$
J\left(\left|u_{k}\right|\right)-J\left(u_{k}\right)=2 \int_{2 m+\varepsilon, k}\left(u_{k}-\left|u_{k}\right|\right) L \dot{\varphi}_{0} \leqq 0
$$


since $L \phi_{0} \geqq 0$ in $\Omega_{m+\varepsilon, \infty}$. By the uniqueness of the minimizing function $u_{k}$ it follows that $u_{k}=\left|u_{k}\right|$ a.e. and consequently $u_{k} \geqq 0$. If $k_{1}>k_{2}>$ $m+2 \varepsilon$ then $u_{k_{2}}-u_{k_{1}}$ is a solution in $H^{1}\left(\Omega_{m+\varepsilon, k_{2}}\right)$ of $L u=0$ and since the the constant function $v \equiv 0$ is also a solution of $L v=0$ then $\max \left(u_{k_{2}}-u_{k_{1}}, 0\right)=\left\{u_{k_{2}}-u_{k_{1}}\right\}^{+}$is a subsolution (in $H_{0}^{1}\left(\Omega_{m+\varepsilon, k_{2}}\right)$ ) by Lemma 2. Consequently,

$$
B\left(\left\{u_{k_{2}}-u_{k_{1}}\right\}^{+}, \dot{\phi}\right) \leqq 0,
$$

for all nonnegative $\phi$ in $C_{0}^{\infty}\left(\Omega_{m+\varepsilon, k_{2}}\right)$ and by continuity:

$$
B\left(\left\{u_{k_{2}}-u_{k_{1}}\right\}^{+},\left\{u_{k_{2}}-u_{k_{1}}\right\}^{+}\right) \leqq 0 .
$$

It follows that $\left\{u_{k_{2}}-u_{k_{1}}\right\}^{+}=0$ and hence $u_{k_{1}} \geqq u_{k_{2}}$.

Lemma 4. Let the conditions of Lemma 3 hold. Then there exists a positive function $u \in C^{2}\left(\Omega_{m+2 \varepsilon, \infty}\right)$ such that $L u(x)=0$ for $x \in$ $\Omega_{m+2 \varepsilon, \infty}$.

Proof. Consider the sequence $\left\{u_{i}\right\}_{i>m+2 \varepsilon}$ with $u_{i}$ set identically equal to zero outside $\Omega_{m+\varepsilon, i}$. This is a minimizing sequence for the functional $J$ in the space formed by completing $C_{0}^{\infty}\left(\Omega_{m+\varepsilon, \infty}\right)$ in the \|\|$_{L^{-}}$ norm since if $\phi \in C_{0}^{\infty}\left(\Omega_{m+\varepsilon, \infty}\right)$ then $J(\phi) \geqq J\left(u_{j}\right)$ for any $j$ chosen so that $\operatorname{supp} \phi \subset \Omega_{m+\varepsilon, j}$. We note that the expression $\|\dot{\phi}\|_{L}^{2}=(\dot{\phi}, L \phi)$ defines a norm even for $\phi \in C_{0}^{\infty}\left(\Omega_{m, \infty}\right)$ since $L$ has no nodal domains in $\Omega_{m, \infty}$. Consequently, the Cauchy-Schwartz inequality shows that the map $\phi \rightarrow$ $\left(\phi, L \dot{\phi}_{0}\right)$ is a bounded linear functional on the space $C_{0}^{\infty}\left(\Omega_{m, \infty}\right)$ (and hence on $C_{0}^{\infty}\left(\Omega_{m+\varepsilon, \infty}\right)$ ), with respect to the \|\|$_{L}$-norm. By means of the Riesz representation theorem, we conclude that the minimum of $J$ is achieved in the completion of $C_{0}^{\infty}\left(\Omega_{m+\varepsilon, \infty}\right)$ (with respect to the \|\|$_{L}$-norm) and it follows that the sequence $\left\{u_{i}\right\}$ converges in the \|\|$_{L}$-norm, [12, Chapter 1]. If $\phi$ denotes any function in $C_{0}^{2}\left(\Omega_{m, \infty}\right)$ we again employ the Cauchy-Schwartz inequality to conclude that $\left\{\left(u_{i}\right.\right.$, $L \phi)\}$ converges. Let $x$ denote any point of $\Omega_{m+\varepsilon, \infty}$. Since $\Omega_{m+\varepsilon, \infty}$ is connected, it is possible to find a finite number of spheres $\left\{S_{i}\right\}_{i=1}^{k}$ such that: $L \dot{\phi}_{0}>0$ in $\bar{S}_{1}$; the center of $S_{i+1}$ belongs to $S_{i} ; x \in S_{k}$; and $\bigcup_{i=1}^{k} \bar{S}_{i} \subset$ $\Omega_{m+\varepsilon, \infty}$. As noted above, the sequence $\left\{\left(u_{i}, L \phi_{0}\right)\right\}$ converges. Since $L \phi_{0} \geqq 0$ in $\Omega_{m+\varepsilon, \infty}$ then $\left\{u_{i}\right\}$ is $L^{1}$ cauchy in $S_{1}$. Let $\phi_{1}$ be a $C_{0}^{2}\left(\Omega_{m, \infty}\right)$ function constructed by the above procedures such that $L \dot{\phi}_{1}>0$ in $\bar{S}_{2}-S_{1}$ and $L \phi_{1} \geqq 0$ in the complement of $S_{1}$. Since:

$$
\left(u_{i}, L \dot{\phi}_{1}\right)=\int_{S_{1}} u_{i} L \phi_{1}+\int_{\Omega-S_{1}} u_{i} L \phi_{1},
$$

we conclude that $\left\{u_{i}\right\}$ is $L^{1}\left(S_{2}\right)$ cauchy. By induction, it follows that $\left\{u_{i}\right\}$ is cauchy in $L^{1}\left(S_{k}\right)$. We set $u=\sup u_{i}$ and conclude that $u$ is 
of class $L_{l o c}^{1}\left(\Omega_{m+\varepsilon, \infty}\right)$ and that for all $\phi \in C_{0}^{\infty}\left(\Omega_{m+\varepsilon, \infty}\right)$ we have $(u, L \phi)=$ $\left(\dot{\phi}, L \dot{\phi}_{0}\right)$. Clearly if supp $\dot{\phi}$ does not intersect $\Omega_{m+\varepsilon, m+2 \varepsilon}$ then $(u, L \dot{\phi})=$ 0 . Let $K$ denote a regular subdomain with $\bar{K}$ a compact subset of $\Omega_{m+\varepsilon, \infty}$ which does not intersect $\Omega_{m+\varepsilon, m+2 \varepsilon}$. Following a standard regularity argument (see, for example, [4, p. 195]), we employ the Sobolev embedding theorem as follows: Let $\phi \in C_{0}^{\infty}(K)$ then:

$$
|(u, \phi)| \leqq \sup _{x \in K^{*}}|\dot{\phi}(x)| \cdot \int_{K}|u| \leqq C\|\dot{\phi}\|_{[n / 2]+1} \cdot \int_{K}|u| \cdot
$$

It follows that the map $\phi \rightarrow(u, \phi)$ is a bounded linear functional on the space $H_{0}^{[n / 2]+1}(K)$. By the Riesz representation theorem we have:

$$
(u, \dot{\phi})=\left(u_{0},(-1)^{[n / 2]+1} \Delta^{[n / 2]+1} \phi\right),
$$

for some $u_{0}$ in $H_{0}^{[n / 2]+1}$, where $H_{0}^{[n / 2]+1}(K)$ is now considered as the completion of $C_{0}^{\infty}(K)$ in the (equivalent) norm:

$$
\|\dot{\phi}\|^{2}=\left(\dot{\phi},(-1)^{[n / 2]+1} \Delta^{[n / 2]+1} \phi\right) .
$$

It follows that:

$$
(u, \phi)=\left(\psi_{0},(-1)^{t} \Delta^{t} \dot{\phi}\right)
$$

and, consequently,

$$
(u, L \dot{\phi})=\left(\psi_{0},(-1)^{t} \Delta^{t} L \phi\right)=0,
$$

for some $\psi_{0}$ in $L^{2}(K)$ and all $\phi$ in $C_{0}^{\infty}(K)$, where $t=[1 / 2[(n+6) / 2]]$. Since the coefficients of $L$ are of class $C^{3 t+1}$ and $C^{3 t}$ respectively, we conclude by a classical result (see, for example, $\left[5\right.$, p. 56]) that $\psi_{0} \in$ $C^{2 t+2}(K)$ and consequently that $u \in C^{2}(K)$. Hence $u$ is a classical solution of $L u=0$ in $\Omega_{m+2 \varepsilon, \infty}$. Since $u$ is obviously nonnegative, then $u$ must be positive [10].

It is interesting to note that the conclusion of Lemma 4 cannot be strengthened to read: "there exists a function $u$ solution of $L u=$ 0 in $\Omega$ and positive in $\Omega_{m+\varepsilon, \infty}$ ", as the following counterexample shows. Let $\Omega=E^{2}$ and let $c$ denote any regular nonpositive function with support in $\{x|| x \mid<1\}$ such that the operator formally defined by $L u=-\Delta u+c u$ has no nodal domains in $\{x|| x \mid<2\}$. $L$ has no nodal domains in $\Omega_{1, \infty}$ since if $\phi \in C_{0}^{\infty}\left(\Omega_{1, \infty}\right)$ then $L \phi=-\Delta \dot{\phi}$. Assume that there exists a function $v$ such that $L v=0$ in $E^{2}$ and $v>0$ in $\Omega_{1+\varepsilon, \infty}$ with $0<\varepsilon<1$. If $v$ vanishes at some point of $E^{2}$ then it must change sign [10]. By Lemma $1,(-v)^{+} \in H_{0}^{1}(\{x|| x \mid<2\})$ and by Lemma $2(-v)^{+}$is a subsolution. Consequently, we have

$$
B\left((-v)^{+},(-v)^{+}\right) \leqq 0 .
$$


If $(-v)^{+} \neq 0$ then we have a contradiction to the assumption that $L$ has no nodal domains in $\{x|| x \mid<2\}$. It follows that $v$ is positive in $E^{2}$ and therefore $\Delta v=c v \leqq 0$. But, by Liouville's theorem, any function which is bounded below and superharmonic in the whole of $E^{2}$ is a constant. If $c$ is nontrivial, this is a contradiction.

THEOREM 1. $L$ is strongly oscillatory iff every $C^{2}$ function $u$, solution of $L u=0$ in some neighborhood of $\infty$, is oscillatory.

Proof. If $L$ is strongly oscillatory then, given any neighborhood $N$ of $\infty$ it follows by the standard theory of eigenvalue problems that there exists a bounded domain $D \subset N \cap \Omega$ and a function $\omega \in$ $H_{0}^{1}(D)$ such that $(\omega, L \omega)<0$. By arguments involving the SwansonPicone identity (see, for example, [14, p. 205], [2]) we conclude that all solutions $u$ of $L u=0$ in $D$ must change sign in $D$. Consequently, all solutions of the equation $L u=0$ in some neighborhood of $\infty$ are oscillatory. Conversely, if $L$ is not strongly oscillatory, then, by Lemma 4 , there exists a positive solution to the equation $L u=0$ in some neighborhood of $\infty$.

The proofs of the lemmas and of Theorem 1 would be even simpler if the fact that $L$ had no nodal domains in $\Omega_{m, \infty}$ implied that, for $\varepsilon>0$, the eigenvalues of $L$ in the bounded subdomains of $\Omega_{m+\varepsilon, \infty}$ were uniformly bounded below by a positive constant. Simple examples can be constructed to show that this is, in general, false.

As an application of Theorem 1 it is possible to give strengthened versions of known nonoscillation theorems. As an example we give the following corollary which strengthens a result of C. A. Swanson [15], which is itself an extension of a result of Glazman.

COROLlaRY 1. Assume that $\Omega$ is the complement of a sphere in $E^{n}$, let $\lambda_{0}>0$ denote the ellipticity constant of $L$ (i.e. $\sum_{i, j=1}^{n} a_{i j}(x) \xi_{i} \xi_{j} \geqq$ $\lambda_{0} \sum_{i=1}^{n} \xi_{i}^{2}$ for all $\left(x, \xi_{1}, \cdots, \xi_{n}\right)$ in $\left.\Omega \times E^{n}\right)$, and let $g(r)$ denote the minimum of $c(x)$ on $\{x|| x \mid=r\}$. If

$$
\liminf _{r \rightarrow \infty} r^{2} g(r)>\frac{-(n-2)^{2} \lambda_{0}}{4},
$$

then there exists a positive solution in a neighborhood of $\infty$ to the equation $L u=0$.

Proof. It is shown in [15] that condition (1) is sufficient for $L$ to have no nodal domains in a neighborhood at $\infty$. The conclusion then follows from Theorem 1.

It is obviously possible to obtain other such results by using 
known nonoscillation criteria, but we do not pursue this point.

In conclusion we note that L. M. Kuks has stated related results which, as given in [11], appear valid only under the implicit assumption that the (open) domain $\Omega$ and the coefficients of $L$ are such that the standard existence and uniqueness theories apply to the whole of $\Omega$. Specifically, it is stated in [11] that a necessary and sufficient condition for the unique solvability of the Dirichlet problem in the subdomains of $\Omega$ is that there exists a positive solution to the inequality $L u \geqq 0$ (cf. Definition 2 and Theorem 3 of [11]). However, this is false for the (open) domain $\Omega$ even if $\Omega$ is bounded and regular, unless some restrictions are placed on the coefficients of $L$ up to the boundary of $\Omega$, as the following example indicates: Let $\Omega=(0,1) \times(0,1)$ and let $L$ be formally given by:

$$
L u=\frac{\partial}{\partial x}\left(\frac{1}{x^{2}} \frac{\partial u}{\partial x}\right)+\frac{\partial}{\partial y}\left(\frac{1}{y^{2}} \frac{\partial u}{\partial y}\right)+2\left[\frac{1}{x^{4}}+\frac{1}{y^{4}}\right] u .
$$

Then $u(x, y)=\left(x^{2}-x / n\right)\left(y^{2}-y / n\right)$ solves $L u=0$ and vanishes on the boundary of $(0,1 / n) \times(0,1 / n)$. Consequently, the Dirichlet problem does not have unique solutions in the subdomains of $(0,1) \times(0,1)$. Yet the function $v(x, y)=x y$ satisfies $L v=0$ and is positive in $\Omega$. Analogous examples are possible for unbounded domains. If the "domain" of [11] is not assumed open, i.e., if $\Omega=Q \cup P$ with $Q$ some open set and $P$ a nonempty subset of the boundary of $Q$, then clearly Theorem 3 of [11] is again false, for if the smallest eigenvalue for $L$ in $Q$ is zero and conditions are sufficiently regular then by the Swanson-Picone identity every solution of the inequality $L u \geqq 0$ must vanish in $\Omega$ even though the Dirichlet problem has a unique solution in each (proper) subdomain of $\Omega$. It follows that, as claimed above, Theorem 3 of [11] is valid (for open sets) only under global regularity assumptions and, consequently, it does not imply Theorem 1 . We state a result which is, in form, a local version of Theorem 3 of [11].

COROLlary 2. There exists an integer $m$ such that the Dirichlet problem for $L$ has a unique (generalized) solution in any bounded subdomain of $\Omega_{m, \infty}$ iff there exists an integer $m^{\prime}$ such that the equation $L u=0$ has a positive solution in $\Omega_{m^{\prime}, \infty}$.

Proof. If there exists a positive solution $u$ to the equation $L u=$ 0 in $\Omega_{m^{\prime}, \infty}$ then by Theorem $1, L$ has no nodal domains in $\Omega_{m^{\prime}, \infty}$. Consequently, the Dirichlet problem for any bounded subdomain of $\Omega_{m^{\prime}, \infty}$ has a unique solution. Conversely, if the Dirichlet problem for $L$ in the bounded subdomains of $\Omega_{m, \infty}$ has a unique solution then $L$ has no nodal domains in $\Omega_{m, \infty}$. The existence of a positive solution in a neighborhood of $\infty$ follows from Theorem 1 . 
If $\Omega$ and the coefficients of $L$ are such that, for some $\varepsilon>0$, it is possible to regularly extend the coefficients of $L$ to the open set $\Omega_{m-\varepsilon, \infty}$ of $E^{n}$ in such a way that the extension has no nodal domains in $\Omega_{m-\varepsilon, \infty}$ then we choose $m^{\prime}=m$ in Corollary 2 and in this case we have:

CoROLlaRy 3. The Dirichlet problem for $L$ has a unique solution in any bounded subdomain of $\Omega_{m, \infty}$ iff there exists a positive solution in $\Omega_{m, \infty}$ to the equation $L u=0$.

Note that if $\bar{\Omega} \neq E^{n}$ we may replace " $\Omega_{m, \infty}$ " by " $\Omega$ " in the statement of Corollary 3 and hence we have, for this special case, an analogue of Theorem 3 of [11] for our unbounded domain. Even though it is easy to give simple sufficient conditions for the extenof $L$, as required in Corollary 3 , to be possible, necessary and sufficient conditions for such an extension are not known to the author at this time.

Added in Proof. The significance to spectral theory of the equivalence of the two types of oscillation has been considered by J. Piepenbrink in his recent paper: "Nonoscillatory Elliptic Equations", J. Differential Equations, 15, 541-550 (1974). Theorem 1 answers in the affirmative the question posed by J. Piepenbrink at the end of his paper.

\section{REFERENCES}

1. W. Allegretto and C. A. Swanson, Oscillation criteria for elliptic systems, Proc. Amer. Math. Soc., 27 (1971), 325-330.

2. W. Allegretto, A comparison theorem for nonlinear operators, Annali Scuola Norm. Sup. Pisa, 25 (1971), 41-46.

3. - Eigenvalue comparison and oscillation criteria for elliptic operators, J. London Math. Soc., 3 (1971), 571-575.

4. L. Bers, F. John, and M. Schecter, Partial Differential Equations, Wiley (Interscience), New York, 1964.

5. A. Friedman, Partial Differential Equations, Holt Rinehart and Winston, New York, 1969.

6. I. M. Glazman, On the negative part of the spectrum of one-dimensional and multi-dimensional differential operators on vector-functions, Dokl. Akad. Nauk SSSR (N.S.), 119 (1958), 421-424.

7. V. B. Headley and C. A. Swanson, Oscillation criteria for elliptic equations, Pacific J. Math., 29 (1968), 501-506.

8. K. Kreith, Oscillation theorems for elliptic equations, Proc. Amer. Math. Soc., 15 (1964), 341-344.

9. - Oscillation Theory, Lecture Notes in Mathematics, Vol. 324, SpringerVerlag, Berlin, 1973.

10. - A remark on a comparison theorem of Swanson, Proc. Amer. Math. Soc., 20 (1969), 549-550.

11. L. M. Kuks, Sturm's theorem and oscillation of solutions of strongly elliptic systems, Soviet Math. Dokl., 3 (1962), 24-27. 
12. S. G. Mikhlin, The Problem of the Minimum of a Quadratic Functional, HoldenDay, San Francisco, 1965.

13. G. Stampacchia, Équations Elliptiques du Second Order à Coefficients Discontinus, Les Presses de l'Université de Montréal, 1966.

14. C. A. Swanson, Comparison and Oscillation Theory of Linear Differential Equations, Mathematics in Science and Engineering, Vol. 48, Academic Press, New York, 1968.

15. - Nonoscillation criteria for elliptic equations, Canad. Math. Bull., 12 (1969), 275-280.

16. C. A. Swanson, Comparison theorems for elliptic differential systems, Pacific J. Math., 33 (1970), 445-450.

Received April 9, 1974 and in revised form August 29, 1974.

UNIVERSity OF Alberta 


\section{PACIFIC JOURNAL OF MATHEMATICS}

\section{EDITORS}

RICHARD ARENS (Managing Editor)

University of California

Los Angeles, California 90024

\section{R. A. Beaumont \\ University of Washington \\ Seattle, Washington 98105}

\section{J. DugundJI}

Department of Mathematics

University of Southern California

Los Angeles, California 90007

D. Gilbarg and J. Milgram

Stanford University

Stanford, California 94305

\section{ASSOCIATE EDITORS}
E. F. BECKENBACH
B. H. NeumanN
F. WOLF
K. YOSHIDA

\section{SUPPORTING INSTITUTIONS}

UNIVERSITY OF BRITISH COLUMBIA
CALIFORNIA INSTITUTE OF TECHNOLOGY
UNIVERSITY OF CALIFORNIA
MONTANA STATE UNIVERSITY
UNIVERSITY OF NEVADA
NEW MEXICO STATE UNIVERSITY
OREGON STATE UNIVERSITY
UNIVERSITY OF OREGON
OSAKA UNIVERSITY

UNIVERSITY OF BRITISH COLUMBIA

UNIVERSITY OF CALIFORNIA

MONTANA STATE UNIVERSITY

NEW MEXICO STATE UNIVERSITY

OREGON STATE UNIVERSITY

OSAKA UNIVERSITY

\author{
UNIVERSITY OF SOUTHERN CALIFORNIA \\ STANFORD UNIVERSITY \\ UNIVERSITY OF TOKYO \\ UNIVERSITY OF UTAH \\ WASHINGTON STATE UNIVERSITY \\ UNIVERSITY OF WASHINGTON \\ AMERICAN MATHEMATICAL SOCIETY \\ NAVAL WEAPONS CENTER
}

The Supporting Institutions listed above contribute to the cost of publication of this Journal, but they are not owners or publishers and have no responsibility for its content or policies.

Mathematical papers intended for publication in the Pacific Journal of Mathematics should be in typed form or offset-reproduced, (not dittoed), double spaced with large margins. Underline Greek letters in red, German in green, and script in blue. The first paragraph or two must be capable of being used separately as a synopsis of the entire paper. Items of the bibliography should not be cited there unless absolutely necessary, in which case they must be identified by author and Journal, rather than by item number. Manuscripts, in triplicate, may be sent to any one of the editors. Please classify according to the scheme of Math. Reviews, Index to Vol. 39. All other communications should be addressed to the managing editor, or Elaine Barth, University of California, Los Angeles, California, 90024.

The Pacific Journal of Mathematics expects the author's institution to pay page charges, and reserves the right to delay publication for nonpayment of charges in case of financial emergency.

100 reprints are provided free for each article, only if page charges have been substantially paid. Additional copies may be obtained at cost in multiples of 50 .

The Pacific Journal of Mathematics is issued monthly as of January 1966. Regular subscription rate: $\$ 72.00$ a year (6 Vols., 12 issues). Special rate: $\$ 36.00$ a year to individual members of supporting institutions.

Subscriptions, orders for back numbers, and changes of address should be sent to Pacific Journal of Mathematics, 103 Highland Boulevard, Berkeley, California, 94708.

PUBLISHED BY PACIFIC JOURNAL OF MATHEMATICS, A NON-PROFIT CORPORATION

Printed at Kokusai Bunken Insatsusha (International Academic Printing Co., Ltd.), 270, 3-chome Totsuka-cho, Shinjuku-ku, Tokyo 160, Japan.

Copyright (C) 1973 by Pacific Journal of Mathematics Manufactured and first issued in Japan 


\section{Pacific Journal of Mathematics \\ Vol. 55, No. $2 \quad$ October, 1974}

Walter Allegretto, On the equivalence of two types of oscillation for elliptic

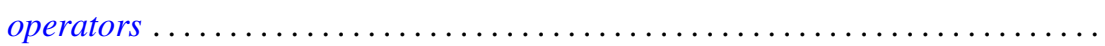

Edward Arthur Bertram, A density theorem on the number of conjugacy classes in

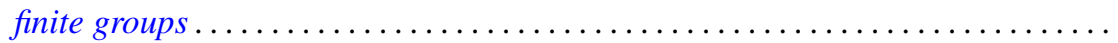

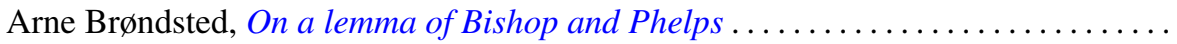

Jacob Burbea, Total positivity and reproducing kernels ..................

Ed Dubinsky, Linear Pincherle sequences . . . . . . . . . . . . . . . . . .

Benny Dan Evans, Cyclic amalgamations of residually finite groups .............

361

Barry J. Gardner and Patrick Noble Stewart, A "going down" theorem for certain

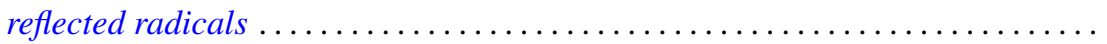

381

Jonathan Light Gross and Thomas William Tucker, Quotients of complete graphs:

revisiting the Heawood map-coloring problem ....................

Sav Roman Harasymiv, Groups of matrices acting on distribution spaces .........

Robert Winship Heath and David John Lutzer, Dugundji extension theorems for

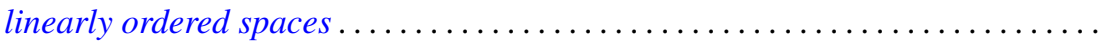

Chung-Wu Ho, Deforming p. l. homeomorphisms on a convex polygonal

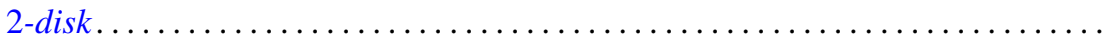

Richard Earl Hodel, Metrizability of topological spaces .................

Wilfried Imrich and Mark E. Watkins, On graphical regular representations of

cyclic extensions of groups .......................... 461

Jozef Krasinkiewicz, Remark on mappings not raising dimension of curves ..... . 479

Melven Robert Krom, Infinite games and special Baire space extensions . . . . . . 483

S. Leela, Stability of measure differential equations . . . . . . . . . . . . . . . . 489

M. H. Lim, Linear transformations on symmetric spaces . . . . . . . . . . . . . . . 499

Teng-Sun Liu, Arnoud C. M. van Rooij and Ju-Kwei Wang, On some group algebra modules related to Wiener's algebra $M_{1} \ldots \ldots \ldots \ldots \ldots \ldots \ldots \ldots \ldots \ldots \ldots$

Dale Wayne Myers, The back-and-forth isomorphism construction ............ 521

Donovan Harold Van Osdol, Extensions of sheaves of commutative algebras by

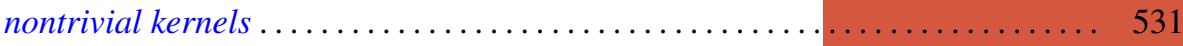

Alan Rahilly, Generalized Hall planes of even order ................... 543

Joylyn Newberry Reed, On completeness and semicompleteness of first countable

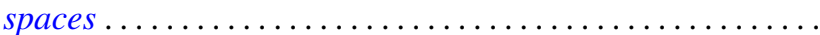

Alan Schwartz, Generalized convolutions and positive definite functions associated

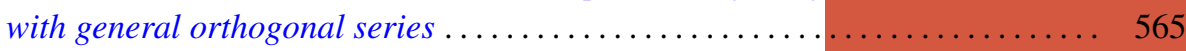

Thomas Jerome Scott, Monotonic permutations of chains . . . . . . . . . . . 583

Eivind Stensholt, An application of Steinberg's construction of twisted groups .... 595

Yasuji Takeuchi, On strongly radicial extensions . . . . ................. 619

William P. Ziemer, Some remarks on harmonic measure in space . . . . . . . . . . 629

John Grant, Corrections to: “Automorphisms definable by formulas” . . . . . . . . 639

Peter Michael Rosenthal, Corrections to: "On an inversion for the general

Mehler-Fock transform pair" ......................... 640

Carl Clifton Faith, Corrections to: "When are proper cyclics injective” . . . . . . 640 